\title{
Carbon Nanostructures as an Electromechanical Bicontinuum
}

\author{
Cristiano Nisoli*, Paul E. Lammert*, Eric Mockensturm ${ }^{\dagger}$ and Vincent H. Crespi* \\ * Department of Physics and Materials Research Institute \\ ${ }^{\dagger}$ Department of Mechanical and Nuclear Engineering \\ The Pennsylvania State University, University Park, PA 16802-6300
}

(Dated: November 2, 2018)

\begin{abstract}
A two-field model provides an unifying framework for elasticity, lattice dynamics and electromechanical coupling in graphene and carbon nanotubes, describes optical phonons, nontrivial acoustic branches, strain-induced gap opening, gap-induced phonon softening, doping-induced deformations, and even the hexagonal graphenic Brillouin zone, and thus explains and extends a previously disparate accumulation of analytical and computational results.
\end{abstract}

PACS numbers: $62.25 .+\mathrm{g}$, 81.05.Tp, 63.22.+m, 77.65.-j, 46.05.+b

Vibrations in carbon nanostructures such as tubes, fullerenes, or graphene sheets [1, 2, 3] have a ubiquitous influence on electronic, optical and thermal response: scattering from optical phonons limits charge transport in otherwise ballistic nanotube conductors 4, 5]; twist deformations gap metallic tubes [6, 7]; ballistic phonons transport heat in nanotubes with great efficiency [8, 9, 10]; resonant Raman spectroscopy can unambiguously identify a tube's wrapping indices $(\mathrm{n}, \mathrm{m})[11,12,13$, 14]; electron-phonon interactions may ultimately limit the electrical performance of graphene [15, 16]. Computationally intensive atomistic models of lattice dynamics often lack simplified model descriptions that can facilitate insight, yet traditional analytical continuum models [1, 2, 17, 18], while very useful and important, cannot describe atomistic phenomena without phenomenological extensions [19, 20, 21]. Although continuum models are restricted to long-wavelength physics, they have been used to describe atomic-scale phenomena in bulk binary compounds by incorporating a separate continuum field for each sublattice [23]: in graphene, two fields are necessary. Here we present an analytical "bicontinuum" model that represents the full atomistic detail of the graphenic lattice, including optical modes, nonlinear dispersion of in-plane phonons, electromechanical effects and even the hexagonal graphenic Brillouin zone, a construct generally held to be exclusively atomistic.

Graphene decomposes into the two triangular sublattices of Fig. 1. We describe in-plane deformations of the sublattices via two fields, $u^{i}(x), v^{i}(x), i=1,2$, and their strain tensors $u^{i j}=\partial^{(i} u^{j)}$ and $v^{i j}=\partial^{(j} v^{i)}$. The density of elastic energy contains direct and cross terms:

$$
V[u, v]=d[u]+d[v]+c[u, v]
$$

Six-fold symmetry of the sublattices implies isotropy of the direct terms 24]:

$$
d[u]=\mu^{\prime} u^{i j} u_{i j}+\frac{\lambda^{\prime}}{2} u_{i}^{i} u_{j}^{j} .
$$

Symmetry dictates the form of the cross term

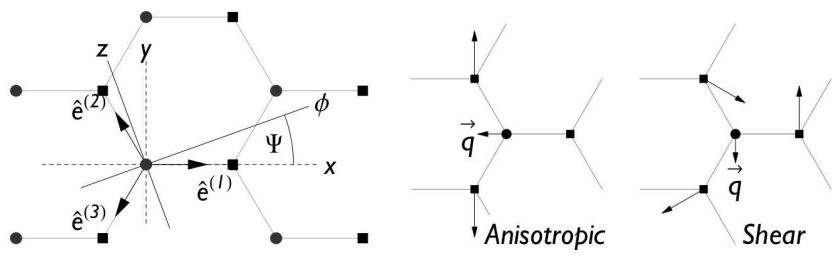

FIG. 1: The two sublattices (circles and squares) of graphene and the three unit vectors $\hat{e}^{(l)}$ used in the text. $\phi, z$ are cylindrical coordinates of a tube, while $\Psi=\pi / 6-\theta_{c}$ with $\theta_{c}$ the chiral angle. Also, anisotropic $\left(u^{x x}=u^{x y}=0, u^{y y}=2 \gamma\right.$, $\left.q^{x}=\ell \gamma\right)$, shear $\left(u^{x x}=u^{y y}=0, u^{x y}=\eta, q^{y}=-\ell \eta\right)$ strains.

$$
\begin{aligned}
c[u, v] & =2 \mu u^{i j} v_{i j}+\lambda u_{i}^{i} v_{j}^{j} \\
& +\alpha(u-v)^{2} \\
& -\beta e_{i j k}\left(u^{i j}+v^{i j}\right)\left(u^{k}-v^{k}\right)
\end{aligned}
$$

The tensor $e_{i j k}$, which is invariant under $C_{3 v}$, can be represented by the three unit vectors $\left\{\hat{e}^{(l)}\right\}$ of Fig. 1

$$
e_{i j k}=\frac{4}{3} \sum_{l=1}^{3} \hat{e}_{i}^{(l)} \hat{e}_{j}^{(l)} \hat{e}_{k}^{(l)} .
$$

Only the last term in Eq. 14 is not invariant under general rotation. (In nanotubes, it depends on the helical angle $\theta_{c}: e_{\phi \phi \phi}=-e_{\phi z z}=-\sin \left(3 \theta_{c}\right), e_{z z z}=-e_{\phi \phi z}=$ $-\cos \left(3 \theta_{c}\right)$, where $\phi, z$ are defined in Fig. 11). This elastic energy density, the lowest-order approximation in both derivatives and fields, contains six parameters: $\mu^{\prime}$ and $\lambda^{\prime}$, being confined to one sublattice, describe next-neighbor interactions; the cross terms $\mu$ and $\lambda$ describe nearestneighbor interaction; $\alpha$ describes the stiffness against relative shifts of the sublattices; $\beta$ determines the strength of rotational symmetry breaking and so carries the point group symmetry of graphene. These parameters are normalized to the sublattice surface density $\sigma_{s}$, so that the elastic energy is $W=\int \sigma_{s} V d^{2} x$.

Taking $\frac{1}{2} \sigma_{s}\left(\dot{u}^{2}+\dot{v}^{2}\right)$ as the surface density of kinetic 
energy, the equations of motion read

$$
\left\{\begin{array}{l}
\ddot{u}^{i}=\partial_{j} \sigma_{(u)}^{i j}-2 \alpha\left(u^{i}-v^{i}\right)+\beta e_{l m}^{i}\left(v^{l m}+u^{l m}\right) \\
\ddot{v}^{i}=\partial_{j} \sigma_{(v)}^{i j}+2 \alpha\left(u^{i}-v^{i}\right)-\beta e_{l m}^{i}\left(v^{l m}+u^{l m}\right)
\end{array}\right.
$$

with the sublattice 2-D stress tensors

$$
\left\{\begin{aligned}
\sigma_{(u)}^{i j} & =2 \mu^{\prime} u^{i j}+\lambda^{\prime} \delta^{i j} u_{k}^{k}+2 \mu v^{i j}+\lambda \delta^{i j} v_{k}^{k} \\
& -\beta e^{i j}{ }_{k}\left(u^{k}-v^{k}\right) \\
\sigma_{(v)}^{i j} & =2 \mu^{\prime} v^{i j}+\lambda^{\prime} \delta^{i j} v_{k}^{k}+2 \mu u^{i j}+\lambda \delta^{i j} u_{k}^{k} \\
& -\beta e^{i j}{ }_{k}\left(u^{k}-v^{k}\right)
\end{aligned}\right.
$$

As expected, $\alpha$ determines the frequency of two degenerate $k=0$ optical modes: $\omega_{\Gamma}^{2}=4 \alpha$.

First, we briefly show that the usual macroscopic elastic energy of graphene and its Lamé coefficients can be obtained from $V$ by considering a static, uniform solution of Eqs. 5 with identical deformations on both lattices with an internal displacement $2 q^{i} \equiv u^{i}-v^{i}$ :

$$
2 q^{i}=\ell e_{l m}{ }^{i} u^{l m}=\ell e_{l m}{ }^{i} v^{l m},
$$

where $\ell=\beta / \alpha$ is a characteristic length. Anisotropic $\left(2 \gamma=u^{x x}-u^{y y}\right)$ and shear $\left(\eta=u^{x y}\right)$ strains produce internal displacements $q^{x}=\ell \gamma$ and $q^{y}=-\ell \eta$ (Fig. 1). The elastic energy for uniform deformations $W_{u}=\int V_{u} \sigma_{g} d^{2} x$ then simplifies to

$$
\begin{aligned}
V_{u}[u, q] & =\left(\mu_{R}+\frac{\beta^{2}}{\alpha}\right) u^{i j} u_{i j}+\frac{1}{2}\left(\lambda_{R}-\frac{\beta^{2}}{\alpha}\right) u_{i}^{i} u_{j}^{j} \\
& +2 \alpha q^{2}-2 \beta e_{i j k} u^{i j} q^{k},
\end{aligned}
$$

where $\sigma_{g}=2 \sigma_{s}=2.26 \mathrm{~g} \mathrm{~cm}^{-2}$ is the surface density of graphene, $\mu_{R} \equiv \mu+\mu^{\prime}-\frac{\beta^{2}}{\alpha}, \lambda_{R} \equiv \lambda+\lambda^{\prime}+\frac{\beta^{2}}{\alpha}$ the measurable Lamé coefficients [24]. Macroscopic problems do not distinguish between the two sublatices; eliminating $q^{i}$ in Eq. 8 through Eqs. 4 and 7 we obtain the familiar, isotropic, macroscopic energy for graphene, $V_{u}=\mu_{R} u^{i j} u_{i j}+\lambda_{R} u_{i}^{i} u_{j}^{j} / 2$. In the long wavelength limit Eqs. 5 returns the familiar longitudinal and transverse speeds of sound in terms of the Lamé coefficients: $v_{L}^{2}=2 \mu_{R}+\lambda_{R}, v_{T}^{2}=\mu_{R}$.

The out-of-plane displacements $u_{\perp}(x)$ and $v_{\perp}(x)$ do not couple with the in-plane $u^{i}, v^{i}$ in the harmonic limit: invariance under simultaneous sign change of $u_{\perp}$ and $v_{\perp}$ prevents it, for flat sheets. Introducing $2 p_{\perp}(x)=u_{\perp}(x)+$ $v_{\perp}(x)$ and $2 q_{\perp}(x)=u_{\perp}(x)-v_{\perp}(x), V_{\perp}$ must be invariant under $p_{\perp} \rightarrow p_{\perp}+L(x), L(x)$ a linear function in the plane, and thus, can contain only second (and higher) derivatives in $p_{\perp}$. Symmetry dictates (cf. Appendix)

$$
\begin{aligned}
V_{\perp} & =4 \alpha_{\perp} q_{\perp}^{2}-4 \alpha_{\perp}^{\prime} \partial_{i} q_{\perp} \partial^{i} q_{\perp}+4 \beta_{\perp} e_{i j k} \partial^{k} q_{\perp} \partial^{i j} p_{\perp} \\
& +2 \mu_{\perp}^{+} \partial_{i j} p_{\perp} \partial^{i j} p_{\perp}+\lambda_{\perp}^{+} \partial_{i}^{i} p_{\perp} \partial_{i}^{i} p_{\perp} \\
& -2 \mu_{\perp}^{-} \partial_{i j} q_{\perp} \partial^{i j} q_{\perp}-\lambda_{\perp}^{-} \partial_{i}^{i} q_{\perp} \partial_{i}^{i} q_{\perp} .
\end{aligned}
$$

The frequency of the $k=0$ out-of-plane optical mode is $2 \sqrt{\alpha_{\perp}}$, and the out-of-plane acoustic branch is quadratic at small wave-vector, as expected.

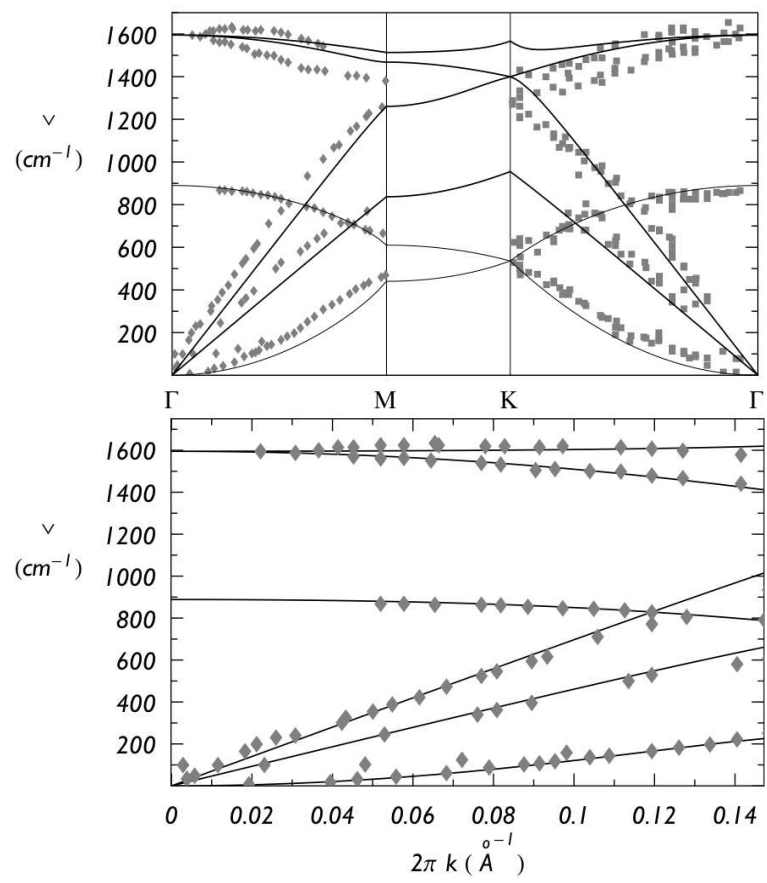

FIG. 2: Bicontinuum phonons compared to EELS data (diamonds [26] and squares [27]), fitting either to the entire Brillouin zone (top) or just around $\Gamma$ along $\Gamma \rightarrow M$.

The bicontinuum phonons are much more richly structured than in a traditional continuum model: they include all the optical branches, show nonlinear dispersion at large wavevector, and even display the main features of the Brillouin zone, all without sacrificing the advantages of a continuum framework. Plane-wave solutions of Eqs. 5 returns an analytically solvable fourth-order secular equation in $\omega(k)$, yielding two acoustic and two optical branches. The longitudinal branches cross at the vertices of a hexagon. Since the two-field elastic energy density respects the point group symmetry of the graphene lattice, this hexagon is oriented just as the graphene Brillouin zone; although the model, unlike in the envelope function approach [25], has no built-in length scale, the elastic parameters can be constrained so that the crossing point coincides with the $K$ point of graphene. A similar argument holds for the out-of-plane modes: strikingly one can construct the correct Brillouin zone within a continuum model. Fig. 2 2 shows the bicontinuum phonons fit to electron-energy-loss spectroscopy (EELS) data [26, 27] for parameters fitted either to the full Brillouin zone or just around $\Gamma$ [28].

The bicontinuum provides a unified framework for nanotube mechanics which can describe all current computational results on the coupling of nanotube phonons to static structural distortions, to each other (e.g. breathing-to-Raman or longitudinal-to-transverse modes in helical tubes) and to the tube electronic structure. In a cylindrical geometry with coordinates $\{r, \phi, z\}$, a 
coupling between the tangential displacements $u^{i}$, and the radial $u^{r}=u_{\perp}$ appears in $V$ of Eq. 1 via $u^{\phi \phi}=$ $\left(\partial_{\phi} u^{\phi}+u^{r}\right) / r$ (and similarly for $v$ ); this accounts for the emergence of the Radial Breathing Mode (RBM) 29]. We consider uniform solutions: $u=u_{o} e^{-i \omega t}, v=v_{o} e^{-i \omega t}$. The tube's helicity can be subsumed into new axes $\{\xi, \zeta\}$ $\left(\xi=\phi \cos 3 \theta_{c}+z \sin 3 \theta_{c} \zeta=-\phi \sin 3 \theta_{c}+z \cos 3 \theta_{c}\right)$ rotated by an angle $3 \theta_{c}$ with respect to the base of the tube. In terms of $p, q$ we obtain $p^{\xi}, p^{\zeta}=0$ and

$$
\left\{\begin{array}{l}
q^{\zeta}\left(\omega^{2}-4 \alpha\right)+2 \frac{\beta}{r} p^{r}=0 \\
p^{r}\left(\omega^{2}-\frac{v_{L}^{2}+\beta^{2} / \alpha}{r^{2}}\right)+2 \frac{\beta}{r} q^{\zeta}=0 \\
q^{\xi}\left(\omega^{2}-4 \alpha\right)=0 \\
q^{r}\left(\omega^{2}-4 \alpha_{\perp}+\frac{2 \mu-2 \mu^{\prime}+\lambda-\lambda^{\prime}}{r^{2}}\right)=0
\end{array}\right.
$$

Unlike standard elasticity [17], which cannot describe optical modes, or standard atomistic descriptions, which cannot be solved analytically, the two-field continuum model enables an exact analytical solution for the coupling between the RBM and the graphite-like optical mode through the first two of Eqs. in (10); the RBM induces a shear in the sublattices, $u^{\phi \phi}=v^{\phi \phi}=u^{r} / r$, which couples with the internal displacement through $\beta$, and vice versa. Thus, the RBM is not purely radial, but has a longitudinal component $q_{B}^{z} \sim \frac{\ell}{2 r} \cos 3 \theta_{c}$, as previously seen in a numerical calculation [30]. Expansion of the RBM frequency in powers of $l / r$ reveals a correction to the the standard continuum result $v_{L} / r$ [17]: $\omega_{B}=\frac{v_{L}}{r}\left[1-\frac{1}{8}\left(\frac{\ell}{r}\right)^{2}+O\left(\frac{\ell}{r}\right)^{4}\right]$. The graphite-like optical modes of chiral tubes are $\omega_{\xi}=\sqrt{4 \alpha}$, $\omega_{\zeta} / \omega_{\xi}=1+\frac{1}{8}\left(\frac{l}{r}\right)^{2}+O\left(\frac{l}{r}\right)^{4}$, also of mixed longitudi$\mathrm{nal} /$ transverse character except for armchair and zig-zag nanotubes, while the out-of-plane optical mode $\omega_{\perp}=$ $\left(4 \alpha_{\perp}-\frac{2 \mu-2 \mu^{\prime}+\lambda-\lambda^{\prime}}{r^{2}}\right)^{1 / 2}$ is purely radial. A density functional theory calculation of the breathing mode 31. reports different frequencies with $\left(\omega_{B}\right)$ and without $\left(\tilde{\omega}_{B}\right)$ coupling to optical modes. We predict $r^{2}\left(\tilde{\omega}_{B}^{2}-\omega_{B}^{2}\right) \rightarrow$ $\beta^{2} / \alpha$ as $r \rightarrow \infty$ : using ref 31] data for $\tilde{\omega}_{B}, \omega_{B}$ we obtain $\ell \equiv \beta / \alpha=0.25 \AA(0.27 \AA)$ for non metallic zig-zag (armchair) tubes, in good agreement with the parameters from our fit to the graphene phonons [28].

The bicontinuum can also describe electron-lattice coupling to both acoustic and optical modes, by incorporating a tight-binding model whose nearest neighbor hopping integrals $t^{(1)}, t^{(2)}, t^{(3)}$ are modulated by the in-plane elastic deformations:

$$
\mathrm{d} t^{(l)}=-\tau \hat{e}_{i}^{(l)} \hat{e}_{j}^{(l)} u^{i j}+\tau \hat{e}_{i}^{(l)} q^{i} / e
$$

where $e$ is the inter-atomic distance and $\tau$ a parameter to be determined [32]. For example, lattice deformations open gaps in metallic tubes, and these gaps in turn affect vibrational frequencies. If $\epsilon_{c}, \epsilon_{v}$ are the conduction and valence bands, we have to nearest neighbors

$$
\epsilon_{c}(k)^{2}-\epsilon_{v}(k)^{2}=\sum_{l} t^{(l)}+2 \sum_{m>l} t^{(l)} t^{(m)} \cos \left(k \cdot a^{(n)}\right),
$$

where $a^{(n)} \equiv e^{(l)}-e^{(m)}, n(l, m)$ is cyclic in $\{1,2,3\}$ (e.g. $\left.a^{(3)} \equiv e^{(1)}-e^{(2)}\right)$ and $\left\{e^{(i)}\right\}$ connects nearest neighbors. From Eqs. 11112 we find the band gap opened by strain in a metallic nanotube to be

$$
\begin{aligned}
\frac{\Delta^{2}}{(3 \tau)^{2}} & =\frac{1}{2} u^{i j} u_{i j}-\frac{1}{4} u_{i}^{i} u_{i}^{i}-\frac{1}{e} e_{i j k} u^{i j} q^{k} \\
& +\frac{1}{e^{2}}\left(\hat{z}_{i} q^{i}\right)^{2}+\frac{1}{e} e_{i j k} \hat{\phi}^{k} u^{i j} \hat{\phi}_{h} q^{h}-\frac{1}{4}\left(e_{i j k} u^{i j} \hat{\phi}^{k}\right)^{2} .
\end{aligned}
$$

In the second line of equation (13) the symmetry of the honeycomb lattice is broken by the unit vectors $\hat{\phi}^{i}, \hat{z}^{i}$ of the cylindrical coordinates. In terms of $2 \gamma^{\prime} \equiv u^{\phi \phi}-u^{z z}$, $\eta^{\prime} \equiv u^{\phi z}, q^{z}$, equation (13) reads

$$
\Delta=3 \tau\left|q^{z} / e+\gamma^{\prime} \cos \left(3 \theta_{c}\right)+\eta^{\prime} \sin \left(3 \theta_{c}\right)\right|,
$$

which corrects and extends a well known previous result within a one-field continuum model [7] that neglected the inner displacement (i.e. $q^{i}=0$ ).

Opening bandgaps in metallic nanotubes causes several shifts in observed quantities. The term proportional to $q_{z}^{2}$ in Eq. (13) show that longitudinal optical modes open a bandgap in metallic tubes of any helicity; the elastic energy lowers by a term proportional to the square of the bandgap, leading to a the softening of longitudinal optical frequency in metallic nanotubes, as revealed by a recent DFT study [33]. Eq. (13) predicts also a softening of the RBM in metallic nanotubes $\frac{\delta \omega_{B}}{\omega_{B}}=-A \cos ^{2}\left(3 \theta_{c}\right)$, highest for zig-zag tubes as seen in DFT [31], and relates it to the optical softening, with $A=(1-\ell / e) \omega_{o p t} \delta \omega_{o p t} e^{2} / 4 v_{L}^{2}$, $\omega_{\text {opt }}$ the graphite-like optical mode, and $\delta \omega_{\text {opt }}$ its softening in metallic tubes $(A \simeq 2 \%)$. Other shifts can be predicted: the speed of sound for the twist mode softens by $\frac{\Delta c_{t}}{c_{t}}=-\frac{v_{L}^{2}}{2 v_{T}^{2}} A \sin ^{2}\left(3 \theta_{c}\right)$, or $\simeq 2.2 \%$ in armchair tubes.

Doping-induced structural deformations can also be studied by minimizing the total energy (elastic plus doped electrons). Subtle phenomena absent in other models 22] can be accessed within the bicontinuum framework. Going to next-nearest-neighbor in the hopping integrals $\left(\mathrm{d} t_{1}^{(l)}=-\tau_{1} \hat{a}_{i}^{(l)} \hat{a}_{j}^{(l)} v^{i j}[32]\right)$, we find that at first order in both $a / r$ and the number of dopant electrons per atom $\rho_{e}$, semiconducting $(n, 0)$ nanotubes show doping-induced changes in tube length $\left(\mathrm{d} L / L=u^{z z}\right)$ and axial bond-length $\left(\mathrm{d} b_{a x}=e u^{z z}-q^{z}\right)$ :

$$
\left\{\begin{array}{l}
\mathrm{d} L / L=\frac{\rho_{e} \tau}{8 m_{C} v_{T}^{2}}\left[ \pm\left(1-\frac{\ell}{e}\right)+\frac{3 \tau_{1}}{2 \tau} \frac{2 \mu_{R}+\lambda_{R}}{\mu_{R}+\lambda_{R}}\right] . \\
\mathrm{d} b_{a x}= \pm \frac{\rho_{e} \tau}{2 m_{C} \omega_{\text {opt }}^{2} e}
\end{array}\right.
$$

where $m_{C}$ is the mass of the carbon atom. The sign is positive (negative) for $r=n \bmod 3=2(n \bmod 3=1)$. 
Recent DFT results [34] indeed show shrinking or stretching of $b_{a x}$ for $n=16,13$ or $n=14,11$ tubes respectively, as predicted by Eq. 15. In DFT, the overall tube lengthens in the second case $(n=14,11)$, again in accord with the bicontinuum; the lengthening found for $r=2$, is less than for $r=1$, perhaps a consequence of the change in sign in Eqs. 15, Finally the shrinking of the axial bond determines an up-shift in the longitudinal graphite-like optical mode and might explain recent Raman results that point toward anomalous bond contraction under doping in semiconducting nanotubes [35, 36].

In summary, a symmetrized two-field continuum model of graphene and carbon nanotubes provides the first unified analytical treatment for a wide range of vibrational and electromechanical phenomena including nonlinear dispersion of in-plane phonons, zone-edge degeneracies and optical modes. A full range of vibrational-electronicmechanical couplings, which were absent from previous continuum models or happened upon in an ad hoc fashion in computational work, can now be understood within a single unified analytical framework. Extending the formalism to include higher-order effects arising from curvature or metallic character (i.e. symmetry breaking terms containing $\hat{\phi}^{i}, \hat{z}^{i}$, as in Eq. 13), anharmonicity (terms higher order in $u^{i j}, v^{i j}$ ), or long-distance interactions (higher partial derivatives) is straightforward. An extension to boron nitride nanotubes, with different coefficients for each sublattice in the direct terms of Eq. 2 . might prove useful to study their piezoelectricity.

\section{Appendix: Derivation of Eq. 3}

The term $c[u, v]$ must be invariant under the combination of $2 \pi / 6$ rotations and the exchange of fields $u \leftrightarrow v$. Adding reflection through the $x$ axis (Fig. 1) then implies $C_{3 v}$ invariance. There is also a field translation invariance: $u(x) \rightarrow u(x)+p, v(x) \rightarrow v(x)+p$. The objects $u^{i}, v^{j}, u^{i j}$, and $v^{i j}$ can be combined pairwise only into tensors of rank two, three and four; thus $c[u, v]$ decomposes into three parts. The first part has terms like $u^{i} v^{j}$; symmetry then implies the form $\alpha(u-v)^{2}$ with $\alpha>0$ to ensure an energy minimum. The second part has terms like $u^{i j} v^{k l}$; the only admissible form is $2 \mu u^{i j} v_{i j}+\lambda u_{i}^{i} v_{j}^{j}$. The third part contains only rank three terms such as $u^{i j} v^{k}$ contracted with a $C_{3 v}$ invariant tensor $e_{i j k}$, giving $e_{i j k} u^{i j} v^{k}$. By requiring invariance under $2 \pi / 6$ rotations conjugated with sublattice switching, and also the field translation invariance, we obtain the form $e_{i j k} u^{i j}\left(u^{k}-v^{k}\right)+e_{i j k}^{*} v^{i j}\left(v^{k}-u^{k}\right)$, where the star means a $2 \pi / 6$ rotation. Since $C_{3 v}$ invariance implies $e_{i j k}^{*}=-e_{i j k}$ we finally obtain the third row of Eq. 3.

[1] S. Iijima, Nature (London) 354, 56 (1991).

[2] R. Saito, G. Dresselhaus and M. S. Dresselhaus, Physical properties of Carbon Nanotubes (Imperial College Press, London 1998).
[3] M. S. Dresselhaus, G. Dresselhaus and P. C. Eklund, Science of Fullerences and Carbon Nanotubes (Academic, New York, 1996).

[4] S. J. Tans et al., Nature 386, 474 (1997).

[5] C. L. Kane et al., Europhys. Lett. 41, 683 (1998).

[6] A. Rochefort, P. Avouris, F. Lesage, D. R. Salahub, Phys. Rev. B 60, 13824 (1999).

[7] L. Yang and J. Han, Phys. Rev. Lett. 85, 154 (2000)

[8] S. Berber, Y-K. Kwon, and D. Tománek, Phys. Rev. Lett. 84, 4613 (2000).

[9] H.-Y. Chiu et al., Phys. Rev. Lett. 95, 226101 (2005).

[10] P. Kim, L. Shi, A. Majumdar and P. L. McEuen Phys. Rev. Lett. 87, 215502 (2001).

[11] E. Richter and K. R. Subbaswamy Phys. Rev. Lett. 79, 2738 (1997).

[12] R. Saito et al., Phys. Rev. B 64, 085312 (2001).

[13] A. Jorio et al., Phys. Rev. Lett. 86, 1118 (2001).

[14] A. Jorio, R. Saito, G. Dresselhaus and M. S. Dresselhaus, Phil. Trans. R. Soc. Lond. A 362, 2311 (2004).

[15] K. S. Novoselov et al., Nature 438, 197 (2005).

[16] S. Y. Zhou et al., Nature Phys. 2, 595 (2006).

[17] G. D. Mahan, Phys. Rev. B 65, 235402 (2002).

[18] H. Suzuura and T . Ando, Phys. Rev. B 65, 235412 (2002); A. Raichura et al., J. Appl. Phys. 94, 4060 (2003). S.V. Goupalov, Phys. Rev. B 71, 085420 (2005)

[19] F. Comas et al., Phys. Rev. B 47, 7602 (1993).

[20] L. Chico and R. Pérez-Álvarez Phys. Rev. B 69, 35419 (2004). L. Chico and R. Pérez-Álvarez, Phys. Rev. B 73, 075425 (2006)

[21] Y. N . Gartstein et al., Phys. Rev. B 68, 115415 (2003)

[22] M. Verissimo-Alves, B. Koiller, H. Chacham, and R. B. Capaz, Phys. Rev. B 67, 161401 (R) (2003).

[23] H. Deresiewicz et al. The collected papers of Raymond D. Mindlin, Springer-Verlag New York (1989).

[24] L. D. Landau, E. M. Lifshitz "Theory of Elasticity" Pergamon Press Oxford (1986). The density of elastic energy for an isotropic system has the form $f=\mu u^{i j} u_{i j}+\frac{\lambda}{2} u_{i}^{i} u_{j}^{j}$, where $\lambda, \mu$ are the Lamé coefficients. Here we renormalize the coefficients to $\sigma_{g}=1$.

[25] B. A. Foreman, Phys. Rev. B 52, 12260 (1995).

[26] C. Oshima et al., Solid State Commun. 65, 1601 (1988).

[27] S. Siebentritt, R. Pues, K. H. Rieder, and A. M. Shikin, Phys. Rev. B 55, 7927 (1997).

[28] The fit around $\Gamma$ returns (in $\mathrm{Km} \mathrm{s}^{-1}$ ) $v_{L}=21, v_{T}=14$, $\left(-2 \mu+2 \mu^{\prime}-\lambda+\lambda^{\prime}+\beta^{2} / \alpha\right)^{1 / 2}=4.4,\left(\mu-\mu^{\prime}\right)^{1 / 2}=$ 15,6 and $\ell \equiv \beta / \alpha=0.3 \AA$ [28]. The fit to the full zone uses $v_{L}=16.5 \mathrm{Km} \mathrm{s}^{-1}, v_{T}=10.8 \mathrm{Km}$ $\mathrm{s}^{-1}, \quad\left(2 \mu-2 \mu^{\prime}+\lambda-\lambda^{\prime}-\beta^{2} / \alpha\right)^{1 / 2}=8.7 \mathrm{Km} \mathrm{s}^{-1}$, $\left(\mu-\mu^{\prime}\right)^{1 / 2}=6,6 \mathrm{Km} \mathrm{s}^{-1}, \ell \equiv \beta / \alpha=0.24 \AA$; an extension to higher derivatives would improve the agreement.

[29] The chiral vector of the tube breaks the hexagonal symmetry and allows for new terms to be introduced in $V$ as curvature corrections, which for simplicity we won't consider here. Different problems will suggest different leading corrections.

[30] E. Dobardžić et al., Phys. Rev. B 68, 045408 (2003).

[31] J. Kürti et al., New J. Phys.5, 125 (2003).

[32] $t^{(l)}, t_{1}^{(l)}$ are the absolute values of the hopping integrals for nearest and next nearest neighbors. We assume they depend only on distance, and thus $\mathrm{d} t=-\tau \mathrm{d} e / e, \mathrm{~d} t_{1}=$ $-\tau_{1} \mathrm{~d} a / a$.

[33] O. Dubay et al., Phys. Rev. Lett. 88, 235506 (2002). 
[34] R. E. Margine et al. submitted to Phys. Rev. Lett.

[35] G. Chen, C. A. Furtado, U. J. Kim, and P. C. Eklund Phys. Rev. B 72155406 (2005).
[36] G. Chen, C. A. Furtado, S. Bandow, S. Iijima and P. C. Eklund Phys. Rev. B 71045408 (2005). 